Revista Brasileira de Farmacognosia Brazilian Journal of Pharmacognosy 21(4): 594-600, Jul./Aug. 2011

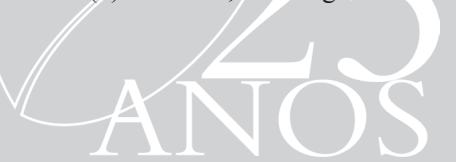

Article

Received 27 Nov 2010

Accepted 18 Jan 2011

Available online 8 Jul 2011

Keywords:

BSA

flavonoids

protein binding

structure-affinity

ISSN 0102-695X

doi: $10.1590 / \mathrm{S} 0102-695 \mathrm{X} 2011005000118$

\section{Molecular structure-affinity relationship of dietary flavonoids for bovine serum albumin}

\author{
Jian Shi, Hui Cao* \\ School of Chemistry and Chemical Engineering, Nantong University, Nantong \\ 226007, Popular Republic of China.
}

\begin{abstract}
The relationship between the molecular structure of dietary flavonoids and their affinities for bovine serum albumin (BSA) were fully investigated by fluorescence titration analysis. The binding process with BSA was significantly affected by the molecular structure of flavonoids under study. The methylation of hydroxyl group in flavonoids enhanced their binding affinities for BSA by 1 to 794 times. Hydroxylation on rings A, B and C also strongly influenced the affinity for BSA. The glycosylation weakened the affinities for BSA by 1-2 orders of magnitude depending on the conjugation site and the class of sugar moiety. The hydrogenation of the $\mathrm{C} 2=\mathrm{C} 3$ double bond slightly increased the binding affinity. The galloylated catechins and pyrogallol-type catechins exhibited higher binding affinities for BSA than non-galloylated and catecholtype catechins, respectively. The affinities for BSA increased with increasing partition coefficients and decreased with increasing hydrogen bond donor and acceptor numbers of flavonoids, which suggested that the binding interaction was mainly caused by hydrophobic forces.
\end{abstract}

\section{Introduction}

Dietary flavonoids are most important polyphenols in plant foods, such as fruits, vegetables, nuts, and tea (Blade et al., 2010; Haghi \& Hatami, 2010; Truchado et al., 2010). Flavonoids are of great interest for their bioactivities, which are basically related to their antioxidative properties (Cohen \& Kennedy, 2010; Côté et al., 2010a; Cheung et al., 2010). The structural differences between the various classes concern the chemistry of the ring $\mathrm{C}$, as well as the number and distribution of hydroxyl groups and their substitutions on the rings A and B (Côté et al., 2010b; Yang et al., 2011). The flavonol moiety, i.e. the 2,3-double bond in conjugation with a 4-oxo group and a 3-hydroxyl group, as well as the 5,7-dihydroxylation at the A-ring has been found to be important structural features for significant antioxidant activity (Morabito et al., 2010). In addition to -OH moieties in the structural arrangements of flavonoids, the resonance of electrons between the rings $\mathrm{A}$ and $\mathrm{B}$ was reported to be essential for the antioxidant and biological activities of the compounds (Natella et al., 2010).

Recently, the interactions between flavonoids and proteins have attracted great interest (Xiao et al., 2007a and 2007b; Xiao et al., 2008; Xiao et al., 2010a and 2010b; Xiao et al., 2011). Most of the reports mainly focused on the binding process and parameters. Few reports, however, have focused on their molecular structure-affinity relationship. Our group has studied the effects of the hydroxylation on ring A of isoflavone (Zhao \& Ren, 2009) and ring B of flavonol (Xiao et al., 2008a), as well as the glycosylation of flavonoids on binding to bovine serum albumin (BSA) (Cao et al., 2009; Xiao et al., 2009). Furthermore, the molecular property-affinity relationship of flavonoids for human serum albumin (HSA) in vitro was described recently (Xiao et al., 2010; Xiao et al., 2011a). However, the comprehensive relationship between the molecular structure of dietary flavonoids and their affinities for BSA was still not understood. In previous reports, appropriate quantities of flavonoids solution were mixed with BSA and subsequently incubated at $300.15 \mathrm{~K}$ for 1 $\mathrm{h}$. Then, the solution was scanned on the fluorometer. Here, the fluorescence titration method was carried out by using trace syringes. The binding affinities obtained by these two methods are certain different. The present work fully investigated the relationship between the molecular structure of dietary flavonoids and their affinities for BSA. Thirty-one flavonoids (Table 1) were studied.

\section{Materials and Methods}

\section{Apparatus and reagents}

The fluorescence spectra were recorded on a JASCO FP-6500 fluorometer (Tokyo, Japan). The 
Table 1. Chemical structures of the various flavonoids and their affinities for BSA in vitro.

\begin{tabular}{|c|c|c|c|c|c|c|}
\hline \multirow{2}{*}{ Subclass } & \multirow{2}{*}{ Name } & \multicolumn{3}{|c|}{ Substitutions } & \multirow[b]{2}{*}{$\lg \mathrm{K}_{\mathrm{a}}$} & \multirow[b]{2}{*}{$\mathrm{n}$} \\
\hline & & $\mathrm{OH}$ & $\mathrm{OCH}_{3}$ & others & & \\
\hline \multirow[t]{10}{*}{ Flavones } & Flavone & & & & 4.79 & 1.032 \\
\hline & 7-OHflavone & 7 & & & 6.10 & 1.252 \\
\hline & Chrysin & 5,7 & & & 4.82 & 0.982 \\
\hline & Baicalein & $5,6,7$ & & & 5.59 & 1.097 \\
\hline & Baicalin & 5,6 & & 7- $\beta$-D-glucuronide & 4.87 & 1.020 \\
\hline & Apigenin & $5,7,4^{\prime}$ & & & 5.69 & 1.106 \\
\hline & Luteolin & $5,7,3^{\prime}, 4^{\prime}$ & & & 5.65 & 1.111 \\
\hline & Wogonin & 5,7 & 8 & & 5.44 & 1.090 \\
\hline & Tangeretin & & $5,6,7,8,4^{\prime}$ & & 4.76 & 0.975 \\
\hline & Nobiletin & & $5,6,7,8,4^{\prime}, 5^{\prime}$ & & 4.77 & 0.968 \\
\hline \multirow[t]{7}{*}{ Flavonols } & Kaempferide & $3,5,7$ & $4^{\prime}$ & & 5.21 & 1.043 \\
\hline & Kaempferol & $3,5,7,4^{\prime}$ & & & 6.35 & 1.221 \\
\hline & Kaempferitrin & $5,4^{\prime}$ & & 3,7-dirhamnoside & 5.09 & 1.082 \\
\hline & Quercetin & $3,5,7,3^{\prime}, 4^{\prime}$ & & & 5.39 & 1.059 \\
\hline & Myricetin & $3,5,7,3^{\prime}, 4^{\prime}, 5^{\prime}$ & & & 5.57 & 1.137 \\
\hline & Galangin & $3,5,7$ & & & 5.39 & 1.078 \\
\hline & Rutin & $5,7,3^{\prime}, 4^{\prime}$ & & 3- $\alpha$-L-Rham-1,6-D-Glc & 5.06 & 1.056 \\
\hline \multirow[t]{6}{*}{ Isoflavones } & Daidzein & $7,4^{\prime}$ & & & 3.23 & 0.765 \\
\hline & Genistein & $5,7,4^{\prime}$ & & & 4.14 & 0.898 \\
\hline & Genistin & $5,4^{\prime}$ & & 7-glucoside & 3.27 & 0.807 \\
\hline & Biochanin A & 5,7 & $4^{\prime}$ & & 5.00 & 1.005 \\
\hline & Tectorigenin & $5,7,4^{\prime}$ & 6 & & 5.41 & 1.087 \\
\hline & Puerarin & $7,4^{\prime}$ & & 8-C-glucose & 3.73 & 0.88 \\
\hline \multirow[t]{2}{*}{ Flavanone } & Naringenin & $5,7,4^{\prime}$ & & & 5.02 & 1.033 \\
\hline & Naringin & $5,4^{\prime}$ & & 7-neohesperidose & 3.55 & 0.814 \\
\hline \multirow[t]{6}{*}{ Flavanonol } & GCG $(2,3$-trans $)$ & $5,7,3^{\prime}, 4^{\prime}, 5^{\prime}$ & & 3-gallate & 4.36 & 0.961 \\
\hline & EGCG $(2,3-c i s)$ & $5,7,3^{\prime}, 4^{\prime}, 5^{\prime}$ & & 3 -gallate & 6.24 & 1.201 \\
\hline & ECG $(2,3-c i s)$ & $5,7,4^{\prime}, 5^{\prime}$ & & 3-gallate & 6.81 & 1.272 \\
\hline & $\mathrm{EC}(2,3-c i s)$ & $3,5,7,4^{\prime}, 5^{\prime}$ & & & & \\
\hline & $\mathrm{EGC}(2,3-c i s)$ & $3,5,7,3^{\prime}, 4^{\prime}, 5^{\prime}$ & & & & \\
\hline & $\mathrm{C}(2,3$-trans $)$ & $3,5,7,4^{\prime}, 5^{\prime}$ & & & & \\
\hline
\end{tabular}

$\mathrm{pH}$ measurements were carried out on a Cole-Parmer PHS-3C Exact Digital pH meter (IL, USA). BSA $(\sim 99 \%$, lyophilized powder) and 7-hydroxyflavone (99.5\%) were purchased from Sigma Co. (MO, USA). Biochanin A, genistein, apigenin, puerarin, catechin (C), epicatechin (EC), and luteolin (99.0\%) were purchased from Aladin Co. Ltd. (Shanghai, China). Flavone, chrysin, and baicalein (99.5\%) were obtained commercially from Wako Pure Chemical
Industries (Osaka, Japan). Kaempferide, kaempferol, tangeretin, nobiletin, quercetin, myricetin, daidzein, baicalin, wogonin, kaempferitrin, galangin, genistin, (-)-epigallocatechin (EGC), (-)-epicatechin gallate (ECG), (-)-epigallocatechin gallate (EGCG), gallocatechin gallate (GCG), and tectorigenin $(>98.0 \%)$ were obtained commercially from Tongtian Co. (Shanghai, China). The working solutions of the flavonoids $\left(1.0 \times 10^{-3} \mathrm{~mol} / \mathrm{L}\right)$ were prepared by 
dissolving each flavonoid with methanol. Tris- $\mathrm{HCl}$ buffer $(0.20 \mathrm{M}, \mathrm{pH} 7.4)$ containing $0.10 \mathrm{~mol} / \mathrm{L} \mathrm{NaCl}$ was selected to keep the $\mathrm{pH}$ value and maintain the ionic strength of the solution. The working solution of BSA $\left(1.0 \times 10^{-5} \mathrm{~mol} / \mathrm{L}\right)$ was prepared with tris $-\mathrm{HCl}$ buffer and stored in refrigerator prior to use. All other reagents and solvents were of analytical grade and all aqueous solutions were prepared using newly doubledistilled water.

\section{Fluorescence spectra}

Fluorescence analysis was performed on a Varian Cray/E spectrofluorophotometer in the ratio mode with temperature maintained by circulating bath. The spectra were recorded in the wavelength range of 310-450 nm upon excitation at $295 \mathrm{~nm}$ when BSA samples were titrated with QDs or flavonoid. Slit widths $(5 \mathrm{~nm})$, scan speed $(240 \mathrm{~nm} / \mathrm{min})$, and excitation voltage $(400 \mathrm{~V})$ were kept constant within each data set and each spectrum was the average of three scans. Quartz cells (1 cm path length) were used for all measurements. Titrations were performed manually by using trace syringes. In each titration, the fluorescence spectrum was collected with the concentrations of BSA at $1.0 \times 10^{-5} \mathrm{~mol} / \mathrm{L}$. The experiments were repeated and found to be reproducible within experimental errors.

\section{Results and Discussion}

The binding constants $\left(K_{d}\right)$ and the number of binding sites ( $n$ )

As representative examples, the fluorescence spectra of BSA after addition of kaempferide are shown in Figure 1 (the fluorescence spectra of BSA quenched by other flavonoids are not given here). In these and all other cases, the fluorescence intensities of BSA decreased remarkably with increasing concentration of flavonoids.

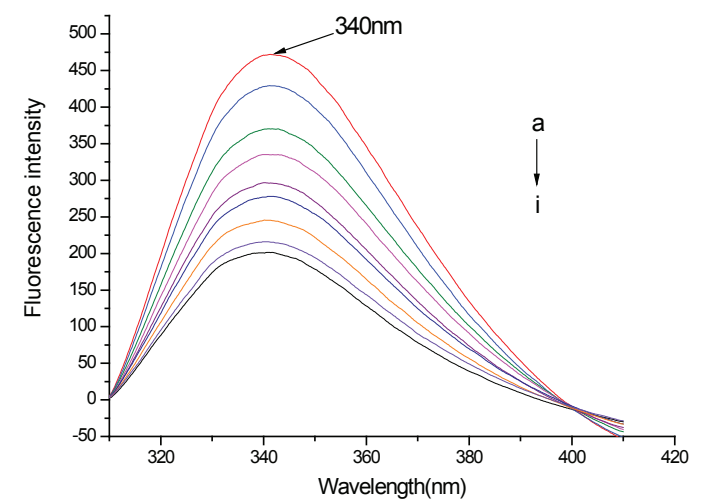

Figure 1. The quenching effect of kaempferide on BSA fluorescence spectra at $300.15 \mathrm{~K} . \lambda_{\text {ex }}=280 \mathrm{~nm}$; BSA, 10.00 $\mu \mathrm{mol} / \mathrm{L}$; a-j: $0.00,1.00,2.00 \ldots 9.00\left(\times 10^{-6} \mathrm{~mol} / \mathrm{L}\right)$ of kaempferide.
The binding constants were calculated according to the double-logarithm equation (Zhao \& Ren, 2009; Xiao et al., 2008b; Cao et al., 2009; Xiao et al., 2009; Xiao et al., 2010c; Xiao et al., 2011b):

$$
\lg \left(F_{0}-F\right) / F=\lg K_{a}+n \lg [Q]
$$

where $F_{0}$ and $F$ represent the fluorescence intensities of BSA in the absence and in the presence of flavonoids, $K_{a}$ is the binding constant, $n$ is the number of binding sites per BSA, and [Q] is the concentration of flavonoids. Table 1 summarizes the results correspondingly calculated results according to equation (1). The values of $\lg \mathrm{K}_{\mathrm{a}}$ are proportional to the number of binding sites (n) (Figure 2), which indicates that the equation (1) used here is suitable to study the interaction between flavonoids and BSA (Berezhkovskiy, 2007; Xiao et al., 2008c).

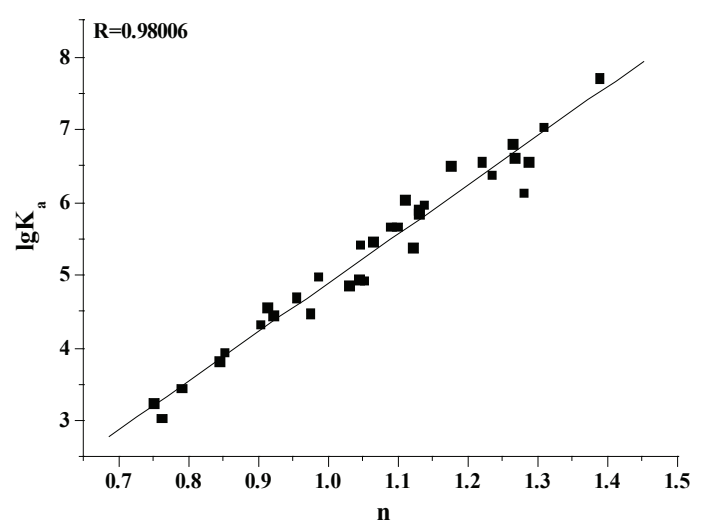

Figure 2. The relationship between the affinities $\left(\operatorname{lgK}_{\mathrm{a}}\right)$ and the number of binding sites ( $\mathrm{n}$ ) between flavonoids and BSA.

Effects of methylation of hydroxyl group in dietary flavonoids on the affinities for BSA

As shown in Figure 3, the methylation of hydroxyl group in flavonoids enhanced the binding affinities for BSA. In general, the methylation of hydroxyl group in flavonoids enhanced their binding affinities for BSA by 1 to 794 times. Extremely, the affinity of daidzein for BSA was found to be 794-time higher than that of formononetin (Figure 3). On the other side, the affinity of nobiletin for BSA was only 1-time higher than that of tangeretin (Figure 3). This result supports that the methylation of flavonoids enhanced the transporting ability, which leads to facilitated absorption and greatly enhanced bioavailability. The methylation of hydroxyl groups in the flavonoids significantly improved their intestinal absorption and metabolic stability (Wen \& Walle, 2006; Walle 2009). Walle found that the methylation seems to be an effective method to enhance the metabolic resistance and transport of flavonoids (Walle 2007). Courts and Williamson concluded that the C-glycoside aspalathin 
was methylated and glucuronidated in vivo (Courts \& Williamson 2009). The potential therapeutic utility of the methylated flavones has also been advanced by finding that the methylation of flavonoids reduces the possibility of toxic side-effects (Walle 2009). The oligomethoxyflavones occurring in plants are less utilized for human consumption compared to the polymethoxyflavones and polyhydroxyflavones.

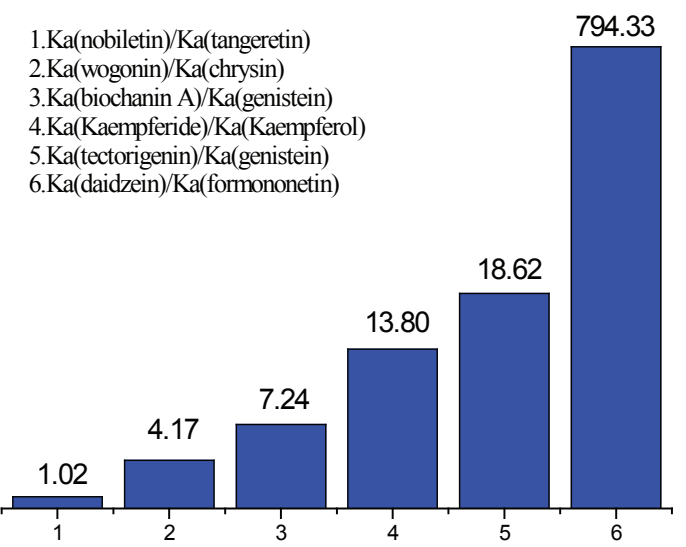

Figure 3. The methoxylation of flavonoids increases the affinity of the flavonoids for BSA.

Effects of hydroxylation of flavonoids on the affinities for $B S A$

Table 2 gave the effects of hydroxylation of flavonoids on the affinities for BSA in vitro. As seen from these data, the hydroxylation on the rings $\mathrm{A}, \mathrm{B}$ and $\mathrm{C}$ of flavonoids dramatically influenced the binding affinities for BSA.

Hydroxylation on ring A of flavones

As illustrated in our recent report (Xiao et al., 2010c), it appears that the optimal number of hydroxyl groups introduced to the ring A of flavones is one for HSA, as the highest binding was observed with 7-hydroxyflavone (containing only one hydroxyl group) (Xiao et al., 2010c). Here, the similar conclusion was found for BSA. 7-hydroxyflavone showed strongest affinity with BSA. However, when more hydroxyl groups are introduced to C-5, C-6, and/or C-7 of flavones, the affinities for BSA slightly decreased.

\section{Hydroxylation on ring $\mathrm{B}$ of flavones}

As shown in Table 2, the binding constants $\left(K_{a}\right)$ between flavones and BSA decreased with the increasing numbers of hydroxyl groups on the B-ring. The hydroxylation on position 4' of flavone significantly improves the binding affinity for BSA. The affinities of apigenin $\left(5,7,3^{\prime}\right)$ for BSA were about 7.41-time higher than that of chrysin $(5,7)$.

\section{Hydroxylation on ring $\mathrm{C}$ of flavones}

As shown in Table 2, the affinities of galangin $(3,5,7)$ for BSA were about 3.72-time higher than that of chrysin $(5,7)$. On the converse, the affinities of apigenin $\left(5,7,3^{\prime}\right)$ and luteolin $\left(5,7,3^{\prime}, 4^{\prime}\right)$ for BSA are about 3.02 and 1.82-time higher than those of kaempferol $\left(3,5,7,3^{\prime}\right)$ and quercetin $\left(3,5,7,3^{\prime}, 4^{\prime}\right)$ for BSA.

\section{Hydroxylation of rings A and B of flavonols}

In this study, it was found that the hydroxylation of rings $\mathrm{A}$ and $\mathrm{B}$ of flavonols slightly affected their binding affinities for BSA. The affinity of quercetin $\left(3^{\prime}, 4^{\prime}\right)$ and myricetin $\left(3^{\prime}, 4^{\prime}, 5^{\prime}\right)$ for BSA was 1.51-time higher than that of kaempferol (4') and quercetin (3', 4'). On the other side, the affinity of galangin (no hydroxyl groups on ring B) for BSA was 1.51-fold higher than that of kaempferol (4'). Flavonols are the most prominent flavonoids in plants. The most prominent flavonols such as quercetin and kaempferol in foods exist with 5,7-dihydroxyl groups on ring A. Here, it was found that the hydroxylation on position 5 of flavonol slightly enhances the binding affinity for BSA by 1.78 times.

\section{Hydroxylation on ring A of isoflavones}

As shown in Table 2, the affinity of genistein for BSA was about 8-time higher than that of daidzein. The affinity of formononetin for BSA was about 13.49time higher than that of biochanin A. with BSA

Comparing the affinities of flavonoid isomers

Previously, we have compared the affinities of flavonoid isomers (apigenin, baicalein, genistein and luteolin, kaempferol) with BSA (Cao et al., 2008). Here, the binding constants $\left(\mathrm{K}_{\mathrm{a}}\right)$ were determined as: apigenin $>$ baicalein $>$ genistein and luteolin $>$ kaempferol. In this study, these results are in good agreement with above data.

\section{Effects of glycosylation of dietary flavonoids on the affinities for BSA}

The dietary flavonoids in nature occur mostly as $\beta$-glycosides. The flavonols are found mainly as the 3- and 7-O-glycoside, although the 4' position may also be glycosylated in some plants (Table 1). Most recently, we have reported the glycosylation of flavonoids lowered the affinity for BSA by 1 to 3 orders of magnitude depending on the conjugation site and the class of sugar moiety (Xiao et al., 2009).

Herein, the effect of glycosylation of dietary 
flavonoids on the affinities for BSA was investigated. In our present study (Figure 4), the glycosylation of flavonoids lowered the affinity for BSA by 1 to 2 orders of magnitude. The affinity of narigenin for BSA was about 29.51-fold higher than that of naringin, but the affinity of kaempferol for BSA is only 1.32-time higher than that of kaempferitrin. The glucopyranosylation (genistin) of genistein lowered the affinity for BSA by 7.41 times. All above, the sugar moieties are in 3 or 7 -positions of flavonoids. The decreasing affinity for BSA after glycosylation may be caused by the nonplanar structure. After the hydroxyl group is substituted by a glycoside, steric hindrance may take place, which weakens the affinity for BSA (Xiao et al., 2009). On the other side, compared with the affinity of daidzein for BSA, the affinity of puerarin (daidzein-8-C-glucose) for BSA increased 3.16 times.

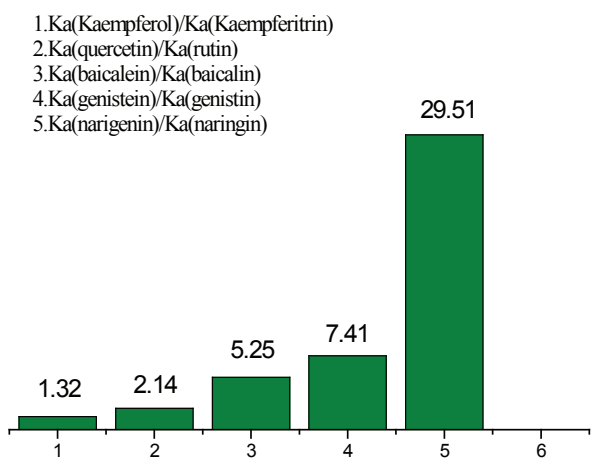

Figure 4. Glycosylation decreases the affinity of the flavonoids for BSA.

Effects of hydrogenation of the $C 2=C 3$ double bond of dietary flavonoids on the affinities for BSA

The $\mathrm{C} 2=\mathrm{C} 3$ double bond in conjugation with a 4-oxo group plays a very important role for the affinity for BSA. It was found that hydrogenation of the $\mathrm{C} 2=\mathrm{C} 3$ double bond of flavonoids increased the binding affinities for BSA. As shown in Table 1, the affinities of apigenin and myricetin for BSA were about 4.68time and 5.25-time higher than those of naringenin and dihydromyricetin, respectively. Hydrogenation of the $\mathrm{C} 2=\mathrm{C} 3$ double bond for many flavonoids increased the binding affinity for BSA by 2-4 orders of magnitude. Planarity of the $\mathrm{C}$ ring in flavonoids maybe important for binding interaction with proteins, as the molecules with saturated $\mathrm{C} 2-\mathrm{C} 3$ bonds (flavanones and certain others) permit more twisting of the $\mathrm{B}$ ring with reference to the $\mathrm{C}$ ring. $\mathrm{A} C 2=\mathrm{C} 3$ double bond increases the $\mathrm{p}$-conjugation of the bond linking the $\mathrm{B}$ and $\mathrm{C}$ rings, which favors near-planarity of the two rings (Edenharder et al., 1993). Molecules with nearplanar structure easier enter the hydrophobic pockets in proteins.

\section{Catechins}

Catechins are the major polyphenols in green tea leaves. The major catechins of green tea extract are (-)-catechin (C), (-)-epicatechin (EC), (-)-epigallocatechin (EGC), (-)-epicatechin gallate (ECG), (-)-epigallocatechin gallate (EGCG), and gallocatechin gallate (GCG). Recent studies have suggested that the catechins form complexes with BSA for transport in blood, and their binding affinity for albumin is believed to modulate their bioavailability. Here, we determined the affinities between catechins and BSA by fluorescence quenching method with double logarithm regression curve. The binding constants $\left(\log 10 \mathrm{~K}_{\mathrm{a}}\right)$ between ECG, EGCG and GCG for BSA were 6.81, 6.24 and 4.36, respectively. However, $\mathrm{EC}, \mathrm{EGC}$, and $\mathrm{C}$ hardly quenched the fluorescence of BSA. It illustrates that the galloylated catechins have higher binding affinities with BSA than non-galloylated catechins and the catechol-type catechins had higher affinities than pyrogallol -type catechins. The presence of the galloyl moiety is the most decisive factor; the increasing hydroxyl groups on ring B decreases the affinity for BSA. In our present study, the affinity of the catechin with 2,3-trans structure (GCG) for BSA was lower than that of the catechin with 2,3-cis structure (EGCG).

Relationship of partition coefficient and the affinity for $B S A$

The lipophilicity of the compounds under study was assessed by their partition coefficient values $\left(\mathrm{XLogP}_{3}\right)$ according to PubChem Public Chemical Database [30]. There is a relationship between the $\mathrm{X}_{\log \mathrm{P}_{3}}$ values and $\lg \mathrm{K}_{\mathrm{a}}$ values for flavonoids (Figure was not shown here). The linear regression equation using the Origin 7.5 software was $\mathrm{X}_{\log } \mathrm{P}_{3}$ $=0.30487+0.28577 \lg \mathrm{K}_{\mathrm{a}} \quad(\mathrm{R}=0.21792)$. From this equation, the binding interaction between flava(o)noids and BSA was slightly caused by hydrophobic forces. BSA consists of large number of $\mathrm{NH}, \mathrm{OH}$ and $\mathrm{COOH}$ groups, which indicates that BSA is a highly polar macromolecule.

To further investigate whether or not the hydrogen bond force plays an important role in binding flavonoids to BSA, the relationships of the hydrogen bond acceptor/donor numbers (N, Data were from reference [30] of flavonoids with the affinities for BSA were shown in Figure 5. The affinities for BSA obviously decreased with increasing hydrogen bond donor and acceptor numbers of flavonoids. These results support the above-mentioned conclusion that the hydrophobic interaction is the main force to bind flavonoids to BSA. 


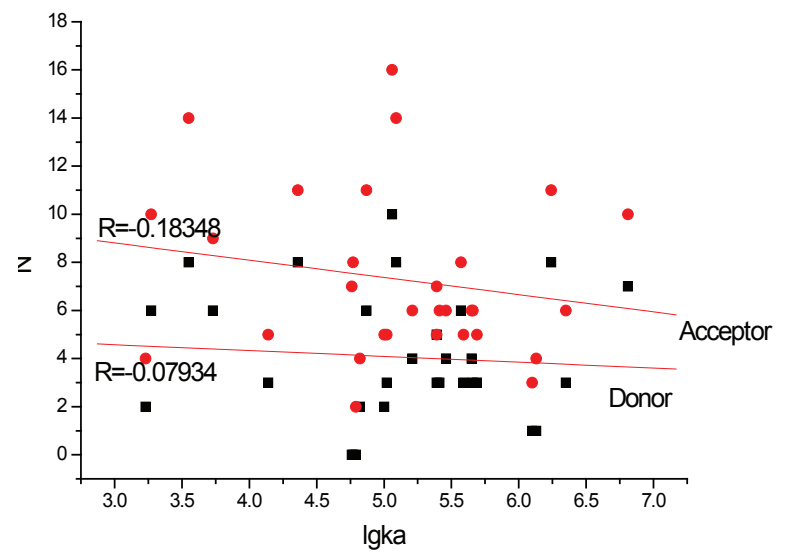

Figure 5. Relationships of the hydrogen bond acceptor/donor number of flavonoids ( $\mathrm{n}$ ) with the affinities for BSA. The hydrogen bond acceptor/donor numbers were taken from PubChem Public Chemical Database (http://pubchem.ncbi. nlm.nih.gov/).

\section{Relationship of topological polar surface area and the} affinity for BSA

The topological polar surface area (TPSA) is defined as the sum of surfaces of polar atoms in a molecule. TPSA has been shown to be a very good descriptor characterizing drug absorption, including intestinal absorption, bioavailability, Caco-2 permeability and blood-brain barrier penetration. Fernandes and Gattass used TPSA to analyze drug transport by multidrug resistance-associated protein 1 (MRP1/ABCC1) (Fernandes \& Gattass 2009). The compounds with high TPSA are transported while those with low TPSA are not. A strong correlation between TPSA and transport properties $\left(K_{m}\right)$ was also found. In our present study, the relationship between TPSA and the binding affinity for BSA was studied. The TPSA values were obtained from PubChem Public Chemical Database (http://pubchem.ncbi.nlm.nih.gov/). There is a relation between the TPSA values and $\operatorname{lgK} \mathrm{a}_{\mathrm{a}}$ values for flavonoids. TPSA values were found to decrease with the increasing $\operatorname{lgK}_{\mathrm{a}}$ for flavonoids (Figure was not shown here). The linear regression equation using the Origin 7.5 software was TPSA $=167.94371-9.35675$ $\operatorname{lgKa}(\mathrm{R}=-0.1383)$. The flavonoids with low TPSA are bound tightly while those with high TPSA are not.

\section{Acknowledgments}

This work was supported by the Main programme of Province Natural Science Foundation of Jiangsu (BK2010034), the Social Development Projects of Nantong (S2009018) and the Science Foundation of Nantong University (09zy004 and 2010K133).

\section{References}

Berezhkovskiy LM 2007. On the calculation of the concentration dependence of drug binding to plasma proteins with multiple binding sites of different affinities: determination of the possible variation of the unbound drug fraction and calculation of the number of binding sites of the protein. J Pharm Sci 96: 249-257.

Blade C, Arola L, Salvado MJ 2010. Hypolipidemic effects of proanthocyanidins and their underlying biochemical and molecular mechanisms. Mol Nutr Food Res 54: 37-59.

Cao H, Wu DH, Wang HX, Xu M 2009. Effect of the glycosylation of flavonoids on interaction with protein. Spectrochim Acta A 73: 972-975.

Cao H, Liu Q, Shi J, Xiao JB, Xu M 2008. Comparing the affinities of flavonoid isomers with protein by fluorescence spectroscopy. Anal Lett 41: 521-532.

Cheung CWY, Gibbons N, Johnson DW, Nicol DL 2010. Silibinin - A promising new treatment for cancer. Anti-cancer Agents Med Chem 10: 186-195.

Cohen SD, Kennedy JA 2010. Plant metabolism and the environment: implications for managing phenolics. Crit Rev Food Sci 50: 620-543.

Côté J, Caillet S, Doyon G, Sylvain JF, Lacroix M 2010a. Analyzing cranberry bioactive compounds. Crit Rev Food Sci 50: 872-888.

Côté J, Caillet S, Doyon G, Sylvain JF, Lacroix M 2010b. Bioactive compounds in cranberries and their biological properties. Crit Rev Food Sci 50: 666-679.

Courts FL, Williamson G 2009. The C-glycosyl flavonoid, aspalathin, is absorbed, methylated and glucuronidated intact in humans. Mol Nutr Food Res 53: 1104-1111.

Edenharder R, von Petersdorff I, Rauscher R 1993. Antimutagenic effects of flavonoids, chalcones and structurally related compounds on the activity of 2-amino-3-methylimidazo[4,5-f]quinoline (IQ) and other heterocyclic amine mutagens from cooked food. Mutat Res 287: 261-274.

Fernandes J, Gattass CR 2009. Topological polar surface area defines substrate transport by multidrug resistance associated protein 1 (MRP1/ABCC1). J Med Chem 52: 1214-1218.

Haghi G, Hatami A 2010. Simultaneous quantification of flavonoids and phenolic acids in plant materials by a newly developed isocratic high-performance liquid chromatography approach. J Agric Food Chem 58: 10812-10816.

Truchado P, Tourn E, Gallez LM, Moreno DA, Ferreres F, Tomás-Barberán FA 2010. Identification of botanical biomarkers in Argentinean Diplotaxis honeys: flavonoids and glucosinolates. J Agric Food Chem 58: $12678-12685$.

Morabito G, Trombetta D, Brajendra KS, Ashok KP, Virinder SP, Naccari C, Mancari F, Saija A, Cristani M, Firuzi O, Saso L 2010. Antioxidant properties of 4-methylcoumarins in vitro cell-free systems. Biochimie 92: 1101-1107.

Natella F, Lorrain B, Prasad AK, Parmar VS, Saso L, Scaccini C 2010. 4-Methylcoumarins as antioxidants: 
Scavenging of peroxyl radicals and inhibition of human low-density lipoprotein oxidation. Biochimie 92: 1147-1152.

Walle T 2009. Methylation of dietary flavones increases their metabolic stability and chemopreventive effects. Int $J$ Mol Sci 10: 5002-5019.

Walle T 2007. Methylation of dietary flavones greatly improves their hepatic metabolic stability and intestinal absorption. Mol Pharmaceut 4: 826-832.

Wen X, Walle T 2006. Methylated flavonoids have greatly improved intestinal absorption and metabolic stability. Drug Metab Dispos 34: 1786-1792.

Xiao JB, Chen JW, Cao H, Ren FL, Yang, CS, Chen Y, Xu M 2007a. Study of the interaction between baicalin and bovine serum albumin by multi-spectroscopic method. J Photochem Photobiol A 191: 222-227.

Xiao JB, Shi J, Cao H, Wu SD, Ren FL, Xu M 2007b. Analysis of binding interaction between puerarin and bovine serum albumin by multi-spectroscopic method. $J$ Pharmaceut Biomed Anal 45: 609-615.

Xiao J, Chen X, Zhang L, Talbot SG, Li GC, Xu M 2008a. Investigation the mechanism of enhanced effect of EGCG on huperzine A inhibiting acetylcholinesterase activity in rats by multi-spectroscopic method. J Agri. Food Chem 56: 910-915.

Xiao, JB, Suzuki M, Jiang XY, Chen XQ, Yamamoto K, Ren FL, Xu M 2008b. Influence of B-ring hydroxylation on interactions of flavonols with bovine serum albumin. $J$ Agric Food Chem 56: 2350-2356.

Xiao JB, Chen XQ, Jiang XY, Hilczer M, Tachiya M 2008c. Probing the interaction of trans-resveratrol with bovine serum albumin: a fluorescence quenching study with Tachiya model. J Fluoresc 18: 671-678.

Xiao JB, Cao H, Wang YF, Zhao JY, Wei XL 2009. Glycosylation of dietary flavonoids decreases the affinities for plasma protein. J Agric Food Chem 57: 6642-6648.
Xiao JB, Chen TT, Chen LS, Cao H, Yang F, Bai YL 2010a. CdTe quantum dots (QDs) improve the affinities of baicalein and genistein for human serum albumin in vitro. J Inorg Biochem 104: 1148-1155.

Xiao JB, Chen LS, Yang F, Liu CX, Bai YL 2010b. Green, yellow and red emitting CdTe QDs decreased the affinities of apigenin and luteolin for human serum albumin in vitro. J Hazard Mater 82: 696-703.

Xiao JB, Cao H, Wang YF, Yamamoto K, Wei XL 2010c. Structure-affinity relationship of flavones on binding to serum albumins: effect of hydroxyl groups on ring A. Mol Nutr Food Res 54: S253-S260.

Xiao JB, Cao H, Chen TT, Yang F, Liu CX, Xu XC 2011a. Molecular property-binding affinity relationship of flavonoids for common rat plasma proteins in vitro. Biochimie 93: 134-140.

Xiao JB, Chen TT, Cao H, Chen LS, Yang F 2011b. Molecular property-affinity relationship of flavanoids and flavonoids for human serum albumin in vitro. Mol Nutr Food Res 55: 310-317.

Yang F, Zhao YR, Kai GY, Xiao JB 2011. Interaction of dietary flavonoids with gamma-globulin: Molecular property-binding affinity relationship aspect. Food Funct 2: 137-141.

Zhao JY, Ren FL 2009. Influence of hydroxylation and glycosylation in ring A of soybean isoflavones on interaction with BSA. Spectrochim Acta A 72: 682685 .

\section{*Correspondence}

Hui Cao

School of Chemistry and Chemical Engineering,

Nantong University

Nantong 26007, PR China

caohui0830@mail.ntu.edu.cn 